\section{Kinasehemmer mit TACE kombinieren?}

\begin{abstract}
Patienten mit inoperablem hepatozellulärem Karzinom profitieren von der transarteriellen Chemoembolisation. Auch der Multikinasehemmer Sorafenib ist bei diesen Patienten effektiv. Ob sich die Kombination dieser Therapieoptionen bewährt, wurde nun untersucht.
\end{abstract}

\begin{abstract}
n Asien wird die transarterielle Chemoembolisation (TACE) als nicht kurative First-Line-Therapie bei großen oder multifokalen Leberzellkarzinomen (intermediäres Stadium B) empfohlen. Metaanalysen bestätigen einen signifikanten Nutzen der TACE, allerdings sind die Langzeitergebnisse unbefriedigend. Daher wurde nun untersucht, ob die gleichzeitige Therapie mit Sorafenib sinnvoll ist. Unter dem Multikinaseinhibitor wurde bei Patienten mit fortgeschrittenem Leberzellkarzinom eine Verlängerung des Gesamtüberlebens nachgewiesen.

An der prospektiven offenen Phase-IIStudie nahmen 165 Patienten aus dem asiatisch-pazifischen Raum mit histolo-
\end{abstract}

gisch oder klinisch diagnostiziertem Leberzellkarzinom im intermediären Stadium teil. Die Behandlung erfolgte mit einer konventionellen TACE und vier bis sieben Tage später mit Sorafenib $(2 \mathrm{x}$ $400 \mathrm{mg} / \mathrm{d}$ ). Die TACE wurde in Form einer selektiven transarteriellen Chemotherapie in den tumorversorgenden Gefäßen mit einer Emulsion aus Lipiodol (5-20 ml) und Doxorubicin $(30-60 \mathrm{mg}$ ) sowie anschließender Embolisation mit absorbierbaren Partikeln durchgeführt. Die Zyklen wurden alle sechs bis acht Wochen wiederholt.

Zwischenergebnisse der Intention-totreat-Analyse mit 147 Patienten zeigen, dass $24,5 \%$ der Patienten ein partielles Ansprechen und $38,8 \%$ eine Stabilisie- rung der Erkrankung erreichten. Die Rate der Krankheitskontrolle betrug $91,2 \%$, die Gesamtansprechrate 52,4\%. Das mediane progressionsfreie Überleben betrug zum Zeitpunkt der Auswertung 270 Tage und die Zeit bis zur Progression 280 Tage. $\mathrm{Zu}$ diesem Zeitpunkt waren noch über $90 \%$ der Patienten am Leben. Durchschnittlich wurden 2,1 Zyklen verabreicht.

Gastrointestinale Nebenwirkungen traten bei 62,6\% der Patienten auf, Hautreaktionen bei $57,8 \%$. Die meisten $\mathrm{Ne}$ benwirkungen waren leicht bis moderat.

Fazit: Die Studie zeigte, dass die Kombination der TACE mit dem Multikinaseinhibitor Sorafenib eine sichere und wirksame Therapie ist, bei der bisher keine unerwarteten Nebenwirkungen auftraten.

Judith Neumaier

Chung Y-H et al. Interim analysis of START: Study in Asia of the combination of TACE (Transcatheter arterial chemoembolization) with sorafenib in patients with Hepatocellular Carcinoma Trial. Int J Cancer. 2013;132(10):2448-58.

\title{
Fischesser haben weniger Leberzellkarzinome
}

\begin{abstract}
Vermehrter Konsum von Fisch und die Vermeidung von rotem bzw. verarbeitetem Fleisch soll das Risiko für einige Krebserkrankungen reduzieren. Dieser Zusammenhang wurde nun für das Leberzellkarzinom untersucht.
\end{abstract}

D ie Omega-3-Fettsäuren im Fisch könnten möglicherweise vor der Entwicklung eines Leberzellkarzinoms schützen, ebenso der hohe Gehalt an Vitamin D und Selen. Im Gegensatz dazu ist der Konsum von vor allem rotem und verarbeitetem Fleisch mit einem erhöhten Krebsrisiko assoziiert. Fleisch könnte über verschiedene Mechanismen auch die Entwicklung eines Leberzellkarzinoms (HCC) fördern.

In der EPIC-Kohorte (European Prospective Investigation into Cancer and Nutrition cohort) wurde nun der Zusammenhang zwischen Fisch- und Fleischkonsum und dem Risiko für die Entwicklung eines HCC untersucht. Bei 191 von 477.206 Teilnehmern wurde zwischen 1992 und 2010 ein Leberzellkarzinom entdeckt. Die Ernährung der Studienteilnehmer wurde mithilfe vali-

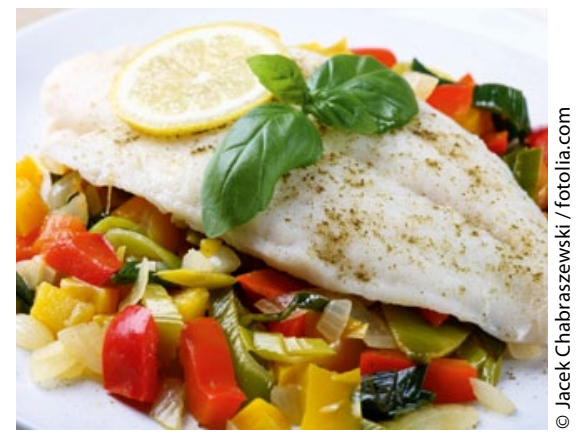

Fischkonsum scheint das Leberkrebsrisiko zu reduzieren.

dierter Fragebögen ermittelt. Außerdem wurden der Hepatitis-B- und -C-Status sowie Leberfunktionsparameter bei einem Teil der Patienten bestimmt.

Binnen durchschnittlich 11,4 Jahren wurden 5.415.385 Personenjahre ausgewertet. Dabei zeigte sich, dass der Ver- zehr von Fisch mit einem niedrigeren HCC-Risiko assoziiert war. Die Hazard Ratio pro Zunahme des Fischkonsums bzw. Abnahme des Fleischkonsums um $20 \mathrm{~g} /$ Tag betrug 0,83 .

Dieser Zusammenhang blieb auch bei Berücksichtigung des $\mathrm{HBV}$ - und $\mathrm{HCV}$ Status und des Leberfunktions-Scores bestehen. Das relative Risiko pro Zunahme des Fischkonsums bzw. Abnahme des Fleischkonsums um 20 g/Tag betrug nun 0,86 . Der Konsum von Fleisch, rotem oder verarbeitetem Fleisch und Geflügel war nicht mit dem Risiko für ein Leberzellkarzinom assoziiert. Die Hazard Ratio betrug 1,16 pro Zunahme des Fleischkonsums und Abnahme des Fischkonsums um 20 g/Tag.

Fazit: In dieser großen europäischen Kohorte war der Fischkonsum mit einem geringerem Risiko für ein Leberzellkarzinom assoziiert.

Judith Neumaier

Fedirko $V$ et al. Consumption of fish and meats and risk of hepatocellular carcinoma: the European Prospective Investigation into Cancer and Nutrition (EPIC). Ann Oncol. 2013 May 12. [Epub ahead of print]. 